\title{
READABILITY OF SERBIAN LAW ON AUDITING
}

\author{
Maša Brkićn ${ }^{*}$, \\ Vule Mizdraković², \\ Blaženka Hadrović Zekić ${ }^{3}$
}

\author{
'Blockx Group, \\ Novi Sad, Serbia \\ ${ }^{2}$ Singidunum University, \\ Belgrade, Serbia \\ ${ }^{3}$ Faculty of Economics in Osijek, \\ Osijek, Croatia
}

\begin{abstract}
:
The main purpose of this paper is to analyse the level of readability of Serbian Law on Auditing. The problem of the research is linguistic understandability concerning comprehension of the text meaning. The readability tests are used as tools in order to get indicators of the readability level. Those tests are Flesch-Kincaid readability tests (The Flesch Reading Ease and Flesch-Kincaid Grade Level), Gunning fog index and SMOG grade. This field is, in most part, unexplored in domestic literature, especially in the domain of texts readability within accounting and auditing. The official translation of national Law on auditing (2013) in the English language has been used in this research. The research results show that Law on Auditing is considered as difficult text to read for average readers. Probably only higher educated readers will be able to grasp the meaning of the text and that limits its use for the wider public.
\end{abstract}

Keywords:

comprehensibility, language, readability index

\section{INTRODUCTION}

Utilization of readability tests can show, in different ways, the easiness or difficulties which may appear in reading and understanding the text. It is usually expressed as a grade level (Hyeoneui, et al., 2007). Readability will be observed from the aspect of comprehensibility of Law on Auditing by potential readers. They can be employees from business entities, banks, insurance companies, audit and accounting companies, government institutions, researchers, professors, students and other parties that are interested in this field. Those users play an active role in maintaining the contemporary economy, education and research. Various methods are applied in assessing the text readability level. Those methods come from psycholinguistics, cognitive linguistics or statistical linguistics. In indicating the readability level, there are a lot of signs which can affect the understanding of the theme. Those signs can be really simple, like sentence length or really complicated, which may lead the reader to the deeper analysis of the sentence and its parts. If complexity of a sentence is higher, there are some time consuming tasks, like calculating all parts of sentences in the texts. (Broda, Ogrodniczuk, Nito 'n, \& Gruszczy'nski, 2014). In all those cases, tests' utilization can improve various analyses, which is a great time saver.

Global use of these formulas started when researchers tested all the formulas in different texts and demonstrated the advantages of analyzing the texts this way. First of all, they can contribute in making the most of the text quality on the level of words and sentences, which can result in reading difficulties. The readability tests can be used for different types of texts in order to provide information about "language style" (is it or is it not difficult for reading). In addition, they can 
help to avoid unnecessary use of scientific jargon, which can easily confuse readers, make the essence of the text difficult to understand or convey the wrong message. In general, we can use readability scores as an objective instrument against confusing writing and incomprehensible texts or assessment tool for determination of the required level of education or age for text comprehension (Woods, Moscardo, \& Greenwood, 1998). The reading level of a text is connected with its usage. If the text is used for „resting" the brain, the reading grade level will be higher than the text whose parts are for classroom use and learning efforts. In other words, the same text will be much easier to understand for the ones with advanced reading skills (with a higher-grade level) and harder for those with average reading skills (lower grade level) (Dubay, 2004).

The audit of financial reports is considered to be a complex process of activities. The main contribution of the audit is an increase of confidence level of financial statements users. Therefore, it can be concluded that audit is a public service. Legal regulation in the field of audit should be easily understandable for all possible stakeholders, who are not necessarily economists. Authors are not familiar with any research in the field of readability assessment of audit text in the Republic of Serbia. This paper contributes to the existing literature in that way. Research conducted for this paper aims to provide the answers regarding the difficulty level of reading the Serbian Law on Auditing. This paper contains the following: after the introduction, through the literature review, we get on to scientific and statistical approaches in this field. Then, we present an overview of the Law on auditing of the Republic of Serbia, which is followed by the evaluation section. In the end, we summarize all the presented options and pay attention to the directions for future researches in this field.

\section{LITERATURE REVIEW}

Readability principals have character which is specified in writing techniques and communication theories. It's the indicator which shows how text can be read and understood. It can be argued that the most important element of written communication, besides the readers' reading skills, is the text readability. That is a text trait that allows certain text to be easier to read, compared to the others. It's often mixed up with "legibility" which refers to layout and typeface (Dubay, 2004). One of the key observations in the oldest researches in this field is that the vocabulary used in the text largely determines its readability. Well-written and readable text heavily depends on the intended audience, which means that personal skills have effect on the quality and length of sentences. Readability researches gave this world the possibility to analyze the texts using readability formulas. Most of those formulas stress education level and
Readability formulas help us to avoid factors that cause difficulty in reading. Improving the text or the words used in the sentences means exceeding reading difficulties. Joann Hackos and Dawn Stephens in Standards for Online Communication (1997) have explained that the best way to avoid reading difficulties is to "conform the accepted style standard" (Dubay, 2004).

Excluding the critics that refer to the validity of traditional readability formulas, they are still used for reading researches. In the past twenty years, technological progress has made way for automation of traditional readability formulas and caused the development of more complex methods for measuring the text difficulty (Benjamin, 2011). Readability formulas are often criticized for their simple formulation and the fact that they do not take into consideration many other variables, which can decrease the readability level or text comprehension. For example, those tests do not have a feedback called "interesting level" which means that material is, or is not, interesting for target audience. Likewise, positive readability score does not necessarily mean that text, in fact, can be well read and understood. One more criticism refers to additional advice and explanations of the way in which we can improve text comprehensibility.

In addition, readability formulas are usually not calculated by hand, instead software programs are used. That raises the question of their consistency in calculation and comparability of the same text reading level. In one of the researches (Mailloux, 1995), four software programs are assessed: Corporate Voice, Grammatix IV, Microsoft Word for Windows and RightWriter. Programs were used on educational text within medical sciences. The same readability formulas that are used in this text were calculated (Flesch-Kincald, Flesch Reading Ease and Gunning Fog index). The results showed that formulas provided significantly different grade equivalent scores; in Microsoft Word program the lowest grade was the most inconsistent (Mailloux, 1995). The same authors found that the average variation equaled 1.3 grade levels between the assessed programs.

On the other hand, numerous studies (Woods, Moscardo, \& Greenwood, 1998) have confirmed positive impact of these tools on text comprehension check. Readability scores are related to:

1. Expecting that readers will read the entire text;

2. The amount of information which readers will remember;

3. The needed time for reading the text and

4. Readers' approaches to difficult texts and their consideration of what is difficult

All readability formulas consider the word length (which means counting the number of characters and number of syllables) and sentence length (counting the number 
of words). The word contains the row of graphic characters that form a semantic unit. The number of characters is a reliable measure of the word length. Words classification (short level words, medium level words, long level words) is specified by readability statistics that show the average length. The word length reflects the degree of reading difficulty. Therefore, words with four or fewer characters are considered as short words and their reading difficulty is low. Medium words will usually have between five and nine characters and their reading difficulty will be moderate. Beyond 9 letters, words will be judged as illegible, or their reading difficulty will be extreme.
The amount of syllables is also an indicator of word length. Namely, short words usually have one syllable and their reading difficulty is considered as low. Medium level words have two syllables and their difficulty is moderate. Beyond three syllables, the word is considered to be complicated and that word is considered as a long word (Fakhfakh, 2015). Readability is certainly connected with sentence length. That length shows the syntactic and semantic difficulty of the text. Most readability formulas are based on the sentence length which can be measured by the number of words. It can predict the degree of reading difficulty.

Table 1. Sentence Length Measured by the Number of Words

\begin{tabular}{ccc}
\hline Linguistic units & Number of words & Level of difficulty \\
\hline Short level sentence & Less than 15 words & Low \\
\hline Medium level sentence & 15 to 20 words & Moderate \\
\hline Long level sentence & More than 20 words & Extreme \\
\hline
\end{tabular}

Source: (Fakhfakh, 2015)

Sentence length and several factors (grammatical and lexical cohesion, complexity of vocabulary, syntactic difficulty) have influence on text understandability. According to linguistic principals, a sentence should not contain more than 15 words. When the number of words in a sentence is increased, the clarity of the sentence is decreased (Fakhfakh, 2015).

Besides vocabulary and sentence structure, some of the main contributors for text comprehension are reader's motivation, prior knowledge and his/hers reading ability. Prior knowledge, as well as text elements, has an effect on retention and documents acceptability. This is the conclusion made in the series of studies in the military (Dubay, 2004). In addition, those studies have found that, while style difficulty seems to have influence on the attention of readers who are inventive regarding material, readers who have wide knowledge of the material may profit a little, if at all, from an easier style of material. These studies have shown difficulties in revealing and measuring the effects of a sentence and other text elements on comprehension, as well. In this regard, it is argued that readability formulas help in overcoming the mentioned problems (Dubay, 2004).

Some researchers concluded that text readability is also related to colors. Comparing the text written on the screen with the text written on paper, authors (Hall \& Hanna, 2003) argued that the text in black and white is hard to read on the computer screen. Human eyes recognize much more easily colored letters on colored background. Taking into account the speed of reading and the number of errors, the optimal screen color will be a little different. Advantage should be given to the next combinations: blue letters on a yellow background, yellow letters on a blue background and red signs on the green background.

To conclude this part of the paper, most authors claim that high text readability will be achieved if the following seven golden rules of writing are followed through (Dubay, 2004):

1. Use short and accustomed words;

2. Avoid the vocabulary specific for a particular profession, for example: computer jargon;

3. Use neutral language regarding culture and gender;

4. Correct use of spelling, grammar and punctuation;

5. Use simple sentences, present tense and avoid the passive voice;

6. It's recommended that a sentence begins with an action verb in imperative mode;

7. Bulleted lists and numbered steps make all information easier to understand.

\section{RESEARCH METHODOLOGY AND RESULTS}

Readability tests and readability formulas are tools that result in an indicator used to evaluate the readability of the text. This estimation is done most often by counting syllables, words and sentences, and the obtained data are included in the appropriate formulas. Several formulas will be used in this text and they will be explained in the text that follows. 
The most common formulas used to estimate readability are Flesch - Kincaid grade level and the Flesch Reading Ease. Those proved to be the most trustworthy, and Flesch Reading Ease is the most commonly tested formula. It is incorporated in the Microsoft Word software because of its ease of use. Using more than one test allows greater access into the text essence. Any measurement is errorsensitive field. Flesch - Kincaid grade level is the most reliable when used with texts for elementary and secondary schools. The Flesch Reading Ease, as well. Some of the formulas can predict higher scores than others, like the SMOG grade (Simple Measure of Gobbledygook) and Gunning Fog index. The SMOG grade predicts the grade level required for 100\% text comprehension. Gunning Fog is widely used in the health care, the Navy and in business publications (Kouame, 2010).

Each of these tests show, in a different way, the required level of education or the age of life for understanding a particular text. They use a few simple factors that are designed to be easily calculated and they are rough approximation of the linguistic factors that determine readability (Pitler \& Nenkova, 2008). First, the results of numerical analysis of Law on auditing on English language are presented in the table that follows.

Table 2. Numerical Analysis of the Text

\begin{tabular}{lc}
\hline Total number of words & 18.900 \\
\hline Total number of characters & 116.829 \\
\hline Total number of syllables & 34.817 \\
\hline Total number of sentences & 1.227 \\
\hline Average length of the sentence & 15.4 words \\
\hline Estimated reading time ${ }^{1}$ & 95 min \\
\hline Estimated speaking time & $152 \mathrm{~min}$ \\
\hline
\end{tabular}

Source: Authors' data

In the next step, previously explained readability indexes are calculated. Flesh-Kincaid Readability Test has two formulas, the Flesh Reading Ease (FRES) and, the Flesh-Kincaid Grade Level (FKGL), both of these formulas take into account the necessary level of education. FRES is calculated according to the following formula:

FRES $=206.835-1.015\left(\frac{\mathrm{N}_{\text {word }}}{\mathrm{N}_{\text {sent }}}\right)-84.6\left(\frac{\mathrm{N}_{\text {syll }}}{\mathrm{N}_{\text {word }}}\right)^{3}$

1 Estimated reading time is based on average reading time of 200 words per minute

2 Estimated speaking time is based on an average reading time of 125 words per minute.

3 Nword - the total number of words in the text, Nsent - the total number of sentences in the text, and Nsyll - the total number of syllables in the text
The higher the value, the material is easier to read, and vice versa. Previous researches have shown that, for example, the texts with the result between 100 and 90 are very easy to read and easily understood by an average 11 -year-old reader. Texts with the result between 70 and 60 seem like plain English texts and they are intended for the public. If the text has a result between 50 and 30 that means that the text is difficult for reading, as well as for understanding. Moreover, the most difficult texts are in the group with results between 30 and 0 . In the case of Law on auditing, the result is 35.4 . Therefore, the text is difficult to read and only higher educated users will understand it.

Flesch-Kincaid grade level (FKGL) is the second type of Flesh-Kincaid readability test and has a wide range of use in the field of education. The result, derived from the following formula, helps professors, teachers, librarians to assess the readability level of various books and texts (Readability formulas, 2017). Values of index between 80 and 100 indicate very easy to easy text to read. Medium readability texts have values of index between 60 and 80 . If the value of index is lower than 30 , it means that the text is very complicated to read, and that university graduates will best understand it. The index value is calculated according to the formula: ${ }^{4} 5$

$$
\text { FKGL }=0.39\left(\frac{N_{\text {word }}}{N_{\text {sent }}}\right)+11.8\left(\frac{N_{\text {syll }}}{N_{\text {word }}}\right)-15.59^{4}
$$

The value of this indicator, in the case of Law on $\mathrm{Au}-$ diting, is 12.2. It means that a highly educated person understands the essence of the text much better than those who do not have a University degree.

The next index used is Gunning Fog Index (GFI). This index can estimate the years of formal education needed for understanding the text after the first reading. The index is used by the writers of the text to check whether it will be comprehensible to the readers it is intended for. Texts for wider public should have an index value of less than 12, while texts that require a comprehensive understanding have an index value higher than 15 . The value of this index is calculated using the formula:

GFI $=0.4\left[\frac{N_{\text {word }}}{N_{\text {sent }}}+100 \frac{N_{\text {cmplxword }}}{N_{\text {word }}}\right]^{5}$

In the case of Law on Auditing, the result is 17.5. High value means that this text is intended for highly educated users or at least college graduates.

4 The same as previous formula.

5 Ncmplxword is the number of complicated words where the word is considered as complicated if it contains three or more syllables. 
Simple Measure of Gobbledygook grade (SMOG) also measures the document readability based on the level of user education. It is most commonly used in the interpretation of materials related to health care that, as is commonly known, are very complicated for understanding (Ley \& Florio, 2007). Results from the SMOG formula point to the following interpretation: grade from 17 to 18 indicates that some text requires higher education for its comprehension. All grades from 19 and more show that a reader must have higher professional qualification for understanding the text essence (MC Laughlin, 1969).
This index is calculated based on the following formula:

$$
\text { SMOG }=1.0430 x \sqrt{N_{\text {cmplxword }} \times \frac{30}{N_{\text {sent }}}+3.1291}
$$

The result is 22.4, which supports the previous results of other formulas. The table number three presents the summary of the calculated formulas.

Table 3. Results of the main readability formulas

\begin{tabular}{ccc}
\hline Reading formula & Results & Readability level \\
\hline FRES & 35.4 & Difficult text \\
\hline FKGL & 12.2 & Very difficult text / Highly educated readers \\
\hline Gunning Fog Index & 17.5 & Very difficult text / Highly educated readers \\
\hline SMOG & 22.4 & Very difficult text / Highly educated readers \\
\hline
\end{tabular}

Source: Authors' data

Taking into account the results obtained in the analysis of the data, it is necessary to understand the fact that, most often, highly educated users have the ability to reasonably understand the essence of the Law. It is assumed that this is due to the manner of formulating articles of the law, using professional terminology and necessary experience in Law, Economy or similar professions.

\section{CONCLUSION}

In this paper the level of readability of Serbian Law on Auditing has been assessed. This text has been selected for the analysis because any employee, student or researcher in the field can read it. Therefore, it is important to maintain high level of understandability in order to provide its implementation. The official translation of the Law on Auditing of the Republic of Serbia has been used. Serbian version of the text has not been used because that prevents the use of readability indexes which are modeled on the text in the English language. Nowadays, the readability formulas are used more than ever. The literacy researches made the writers aware of the limited abilities of their readers and at the same time helped them in writing texts of higher quality. Many factors can affect the reader's comprehension of the text. When used properly, readability formulas can help in increasing chances of successfully transmitting the writer's message in terms of the correct-text-understanding (Dubay, 2004). That is the main reason why these formulas are used in this paper. The results of the analysis show that the text consists of over one thousand sentences and it takes an average reader about an hour and a half to read it. Most commonly used readability indices have been used and the results show that readability level of national Law on Auditing is low. The reader has to be an older, highly educated person in order to fully comprehend the whole text and its sense. The future research will focus on the comparison of the readability indices values for different types of texts (scientific papers, books, newspapers, etc.).

\section{LITERATURE}

Benjamin, R. G. (2011). Reconstructing Readability: Recent Developments and Recommendations in the Analysis of Text Difficulty. Educational Psychology Review (pp. 63-88). University of Georgia, Athens, USA.

Dubay, W. (2004). The Principles of Readability. Retrieved from ResearchGate: https://www.researchgate.net/publication/228965813_The_Principles_of_Readability

Pitler, E., \& Nenkova, A. (2008). Revisiting Readability: A Unified Framework for Predicting Text Quality. Conference on Empirical Methods in Natural Language Processing, EMNLP 2008, (pp. 25-27). Honolulu, Hawaii, USA.

Broda, B., Ogrodniczuk, M., Niton, B., \& Gruszczynski, W. (2014). Measuring Readability of Polish Texts: Baseline Experiments. 9th International Conference on Language Resources and Evaluation (LREC 2014), (pp. 573-580). Reykavik, Iceland. 
Fakhfakh, M. (2015). The readability of international illustration of auditor's report: An advanced reflection on the compromise between normative principles and linguistic requirements. Journal of Economics, Finance and Administrative Science, 21-29.

Hall, R. H., \& Hanna, P. (2003). The Impact of Web Page TextBackground Color Combinations on Readability, Retention, Aesthetics, and Behavioral Intention. Behavior and Information Technology.

Hyeoneui, K., Goryachev, S., Rosemblat, G., Browne, A., Keselman, A., \& Zeng-Treitler, Q. (2007). Beyond Surface Characteristics: A new Health Text-Specific Readability Measurement. AMIA 2007 Symposium Proceedings, (p. 418). Bethesda, Maryland, USA.

Kouame, J. B. (2010). Using Readability Tests to Improve the Accuaracy of Evaluation Documents Intended for LowLiterate Participants. Journal of MultiDisciplinary Evaluation, 132-139.
Ley, P., \& Florio, T. (2007). The use of readability formulas in health care. Psychology, Health \& Medicine, 7-28. Retrieved from Taylor \& Francis Online: http://www. tandfonline.com/doi/abs/10.1080/13548509608400003

Mailloux, S., Johnson, M., Fisher, D. and Pettibone, T. (1995) How Reliable is Computerized Assessment of Readability? Computers in Nursing, 13, (5), pp. 221-225

MC Laughlin, G. H. (1969). SMOG Grading - a New Readability Formula. Journal of Reading, 639 - 646.

Readability formulas. (2017). Retrieved from http://www. readabilityformulas.com/flesch-grade-level-readabilityformula.php

Tarasov, D. A., \& Sergeev, P. A. (2013). The leading as a factor of readability: development of the methodology for educational use. International Conference on $\mathrm{New} \mathrm{Ho-}$ rizons in Education (pp. 2914-2920). TASET, Sakarya University, Turkey.

Woods, B., Moscardo, G., \& Greenwood, T. (1998). A Critical Review of Readability and Comprehensibility Tests. The Journal of Tourism Studies, 49-61. 